\title{
Quantum cloning of orthogonal qubits
}

\author{
J. Fiurášek, ${ }^{1}$ S. Iblisdir, ${ }^{2}$ S. Massar, ${ }^{3}$ and N.J. Cerf ${ }^{2}$ \\ ${ }^{1}$ Department of Optics, Palacký University, 17. listopadu 50, 77200 Olomouc, Czech Republic \\ ${ }^{2}$ Ecole Polytechnique, CP 165, Université Libre de Bruxelles, B-1050 Bruxelles, Belgium \\ ${ }^{3}$ Service de Physique Théorique, CP 225, Université Libre de Bruxelles, B-1050 Bruxelles, Belgium
}

\begin{abstract}
An optimal universal cloning transformation is derived that produces $M$ copies of an unknown qubit from a pair of orthogonal qubits. For $M>6$, the corresponding cloning fidelity is higher than that of the optimal copying of a pair of identical qubits. It is shown that this cloning transformation can be implemented probabilistically via parametric down-conversion by feeding the signal and idler modes of a nonlinear crystal with orthogonally polarized photons.
\end{abstract}

In contrast to classical information, quantum information cannot be copied. This so-called no-cloning theorem [1], which is a direct consequence of the linearity of quantum theory, states that it is impossible to prepare several exact copies (or clones) of an unknown quantum state $|\psi\rangle$. Although exact cloning is forbidden, one can design various quantum cloning machines which produce approximate clones. In particular, much attention has been devoted to the optimal universal cloning machines for qubits, which prepare $M$ identical approximate clones out of $N$ replicas of an unknown qubit, and such that the fidelity of the clones is state-independent 2. Cloning machines for states in a $d$-dimensional Hilbert space (qudits) were also investigated [3], as well as continuousvariable cloning machines for coherent states [4].

In the limit of an infinite number of clones, the optimal cloning reduces to the optimal quantum measurement. In this context, a very interesting observation has been made by Gisin and Popescu [5] who noted that the information about a direction in space is better encoded into two orthogonal qubits than in two identical ones. If we possess a two-qubit state $\left|\psi, \psi_{\perp}\right\rangle$ with $\left\langle\psi \mid \psi_{\perp}\right\rangle=0$, then we can estimate $|\psi\rangle$ with a fidelity $F_{\perp}=(1+1 / \sqrt{3}) / 2 \approx 0.789$ [5, 6]. This slightly exceeds the fidelity of the optimal measurement on a qubit pair $|\psi, \psi\rangle, F_{\|}=3 / 4$. A similar situation occurs for continuous quantum variables. Suppose we want to encode a (randomly chosen) position in phase space. A possible strategy would be to prepare a pair of coherent states $|\alpha, \alpha\rangle$, where the real and imaginary parts of the complex number $\alpha$ represent the phase-space coordinates. However, it is actually better to supply a state $\left|\alpha, \alpha^{*}\right\rangle$ from which $\alpha$ can be inferred via optimal measurement with a lower error [7]. It can also be shown that the state $\left|\alpha, \alpha^{*}\right\rangle$ gives an advantage when cloning coherent states: $M$ identical approximate clones of a coherent state $|\alpha\rangle$ can be prepared with a higher fidelity from the state $\left|\alpha, \alpha^{*}\right\rangle$ than from $|\alpha, \alpha\rangle[\beta]$.

Motivated by this result, we were led to ask whether a similar situation might also occur for qubits. Can $M$ clones of qubit $|\psi\rangle$ be produced from an orthogonal qubit pair $\left|\psi, \psi_{\perp}\right\rangle$ with a higher fidelity than from an identical pair $|\psi, \psi\rangle$ ? In this Letter, we answer this question by an affirmative. We present a universal cloning machine acting on an orthogonal qubit pair that approximately implements the transformation $|\psi\rangle\left|\psi_{\perp}\right\rangle \rightarrow|\psi\rangle^{\otimes M}$ with the optimal fidelity. Then, we show that this cloning transformation can be implemented probabilistically in quantum optics by use of parametric down-conversion. Our proposed setup extends the scheme of Simon et al. [9] by feeding both the signal and idler modes of a nonlinear crystal with $|\psi\rangle$ and $\left|\psi_{\perp}\right\rangle$, respectively.

Let us first provide a simple argument on why we can expect the state $\left|\psi, \psi_{\perp}\right\rangle$ to be better cloned than $|\psi, \psi\rangle$. If we perform an optimal measurement of $\left|\psi, \psi_{\perp}\right\rangle$, we can prepare $M$ identical clones of $|\psi\rangle$, each with a fidelity $F_{\perp}$. In contrast, the fidelity of the optimal universal cloning machine that prepares $M$ clones from a two-qubit state $|\psi, \psi\rangle$ is given by $F_{\|}(M)=(3 M+2) /(4 M)$ [2]. Clearly, $F_{\|}(M)<F_{\perp}$ for sufficiently large $M$. Hence, this (nonoptimal) measurement-based cloning of $\left|\psi, \psi_{\perp}\right\rangle$ is better than the standard cloning of $|\psi, \psi\rangle$.

Let us now seek for a unitary transformation which optimally approximates the transformation $|\psi\rangle\left|\psi_{\perp}\right\rangle \rightarrow$ $|\psi\rangle^{\otimes M}$. Since the set of all states of the form $|\psi\rangle\left|\psi_{\perp}\right\rangle$ span the whole Hilbert space of two qubits, the most general transformation is of the form:

$$
|i\rangle|j\rangle|R\rangle \rightarrow \sum_{k=0}^{M}|M, k\rangle\left|R_{i j k}\right\rangle \quad i, j=0,1
$$

where $|R\rangle$ and $\left|R_{i j k}\right\rangle$ respectively denote the initial and final states of the ancilla, while $|M, k\rangle(k=0, \ldots, M)$ denotes a symmetric $M$-qubit state with $k$ qubits in state $|0\rangle$ and $M-k$ qubits in state $|1\rangle$. The arbitrary state of a qubit $|\psi\rangle$ can be conveniently written as $|\psi\rangle=d(\Omega)|0\rangle=$ $\sum_{i} d_{i 0}(\Omega)|i\rangle$, where the matrix $d(\Omega)$ is given by

$$
d(\Omega)=\left(\begin{array}{cc}
\cos \frac{\vartheta}{2} & e^{-i \phi} \sin \frac{\vartheta}{2} \\
e^{i \phi} \sin \frac{\vartheta}{2} & -\cos \frac{\vartheta}{2}
\end{array}\right),
$$

with $\vartheta$ and $\phi$ denoting the usual polar and azimuthal angles pointing in direction $\Omega$. The linearity of (1) implies that an arbitrary pair of orthogonal qubits transforms according to

$$
|\psi\rangle\left|\psi_{\perp}\right\rangle \rightarrow\left|\Psi_{\text {out }}(\psi)\right\rangle=\sum_{i j k} d_{i 0}(\Omega) d_{j 1}(\Omega)|M, k\rangle\left|R_{i j k}\right\rangle .
$$

We will measure the quality of the transformation by the average single-clone fidelity $F_{\perp}(M)$. Denoting by $\operatorname{Tr}_{1 \text {,anc }}$ 
the partial trace over the ancilla and all the clones but the first one, we get

$$
\begin{aligned}
F_{\perp}(M) & =\int d \Omega\left\langle\psi\left|\operatorname{Tr}_{1, \text { anc }}\left[\left|\Psi_{\text {out }}(\psi)\right\rangle\left\langle\Psi_{\text {out }}(\psi)\right|\right]\right| \psi\right\rangle \\
& =\sum_{i^{\prime} j^{\prime} k^{\prime}} \sum_{i j k}\left\langle R_{i^{\prime} j^{\prime} k^{\prime}} \mid R_{i j k}\right\rangle A_{i j k}^{i^{\prime} j^{\prime} k^{\prime}}
\end{aligned}
$$

where

$$
\begin{aligned}
& A_{i j k}^{i^{\prime} j^{\prime} k^{\prime}}=\sum_{n, n^{\prime}}\left\langle n^{\prime}\left|\operatorname{Tr}_{1}\left[|M, k\rangle\left\langle M, k^{\prime}\right|\right]\right| n\right\rangle \\
& \times \int d \Omega d_{n 0}(\Omega) d_{n^{\prime} 0}^{*}(\Omega) d_{i 0}(\Omega) d_{j 1}(\Omega) d_{i^{\prime} 0}^{*}(\Omega) d_{j^{\prime} 1}^{*}(\Omega) .
\end{aligned}
$$

The coefficients $A_{i j k}^{i^{\prime} j^{\prime} k^{\prime}}$ can be considered as matrix elements of an operator $A$ acting on the space $\mathcal{H} \otimes \mathcal{K}$, where $\mathcal{H}$ denotes the Hilbert space of the two input qubits and $\mathcal{K}$ denotes the Hilbert space of symmetric states of $M$ output qubits. Similarly, $\chi_{i j k}^{i^{\prime} j^{\prime} k^{\prime}}=\left\langle R_{i j k} \mid R_{i^{\prime} j^{\prime} k^{\prime}}\right\rangle$ define matrix elements of an operator $\chi$ also acting on $\mathcal{H} \otimes \mathcal{K}$. The formula (4) for the fidelity thus simplifies to $F_{\perp}(M)=\operatorname{Tr}_{\mathcal{H}, \mathcal{K}}[\chi A]$. The operator $\chi$ uniquely represents the completely positive cloning map, which transforms operators supported on $\mathcal{H}$ onto operators supported on $\mathcal{K}$. By definition, the operators $A$ and $\chi$ are Hermitian and positive semidefinite, $A \geq 0$ and $\chi \geq 0$.

Of course, the transformation (11) should be unitary, which reads $\sum_{k}\left\langle R_{i^{\prime} j^{\prime} k} \mid R_{i j k}\right\rangle=\delta_{i^{\prime} i} \delta_{j^{\prime} j}$. This is equivalent to $\operatorname{Tr}_{\mathcal{K}}[\chi]=\mathbb{1}_{\mathcal{H}}$, where $\mathbb{1}_{\mathcal{H}}$ is the identity operator on $\mathcal{H}$. Thus, introducing a set of Lagrange multipliers $\lambda_{i j}^{i^{\prime} j^{\prime}}$ for these unitarity constraints, our problem amounts to extremize the quantity $W=\operatorname{Tr}_{\mathcal{H}, \mathcal{K}}[(A-\Lambda) \chi]$ under the constraint $\chi \geq 0$, where $\Lambda=\lambda \otimes \mathbb{1}_{\mathcal{K}}$ and $\lambda$ is the matrix of Lagrange multipliers $\left(\mathbb{1}_{\mathcal{K}}\right.$ is the identity operator on $\mathcal{K})$. Varying $W$ with respect to the eigenstates of the operator $\chi$, we get the extremal equation

$$
(A-\Lambda) \chi=0
$$

for the optimal $\chi$. Following [10], this equation can be further transformed into a form suitable for numerical calculation via repeated applications of

$$
\chi=\Lambda^{-1} A \chi A \Lambda^{-1}, \quad \lambda=\left(\operatorname{Tr}_{\mathcal{K}}[A \chi A]\right)^{1 / 2} .
$$

Note that the matrix $\lambda \geq 0$ is determined from the unitarity constraints.

By numerically solving Eq. (7) for $M=2, \ldots 15$, we have been able to conjecture the general analytical form of the optimal transformation:

$$
\left|\psi, \psi_{\perp}\right\rangle \rightarrow \sum_{j=0}^{M} \alpha_{j, M}\left|(M-j) \psi, j \psi_{\perp}\right\rangle \otimes\left|(M-j) \psi_{\perp}, j \psi\right\rangle
$$

where

$$
\alpha_{j, M}=(-1)^{j}\left[\frac{1}{\sqrt{2(M+1)}}+\frac{\sqrt{3}(M-2 j)}{\sqrt{2 M(M+1)(M+2)}}\right]
$$

with $\left|j \psi,(M-j) \psi_{\perp}\right\rangle$ denoting a totally symmetric state of $M$ qubits where $j$ qubits are in state $|\psi\rangle$ and $M-$ $j$ qubits are in state $\left|\psi_{\perp}\right\rangle$. The first $M$ output qubits contain the clones of state $|\psi\rangle$ while the other $M$ qubits contain the clones of $\left|\psi_{\perp}\right\rangle$ (or anticlones).

We stress here that the cloning transformation $(\beta)$ is unitary. Since this is by no means obvious from (8), let us present a proof of this. We can expand any state $\left|j \psi,(M-j) \psi_{\perp}\right\rangle$ in the basis $|M, k\rangle$ as

$$
\left|j \psi,(M-j) \psi_{\perp}\right\rangle=\sum_{k=0}^{M} e^{i(j-k) \phi} D_{k j}^{M}(\vartheta)|M, k\rangle
$$

We will not need an explicit expression for the functions $D_{k j}^{M}(\vartheta)$ here, but will only use some of their properties. Since the functions $D_{k j}^{M}(\vartheta)$ are elements of a (real) unitary matrix, they satisfy the orthogonality relation,

$$
\sum_{j=0}^{M} D_{k j}^{M}(\vartheta) D_{l j}^{M}(\vartheta)=\delta_{k l}
$$

We will also use the following recurrence formula [1],

$$
(2 j-M) D_{k j}^{M}(\vartheta)=(2 k-M) \cos \vartheta D_{k j}^{M}(\vartheta)+\sin \vartheta \sqrt{(k+1)(M-k)} D_{k+1, j}^{M}(\vartheta)+\sin \vartheta \sqrt{k(M-k+1)} D_{k-1, j}^{M}(\vartheta) .
$$

For the purposes of the proof it is convenient to apply a unitary transformation $U_{0}$ on the last $M$ qubits at the output of the cloner and get the state $\left|\Phi_{\text {out }}(\psi)\right\rangle=$ $I^{\otimes M} \otimes U_{0}^{\otimes M}\left|\Psi_{\text {out }}(\psi)\right\rangle$. The unitary transformation $U_{0}$ flips the states $|0\rangle$ and $|1\rangle,|0\rangle \rightarrow|1\rangle$ and $|1\rangle \rightarrow-|0\rangle$. Thus $\left|(M-j) \psi_{\perp}, j \psi\right\rangle \rightarrow(-1)^{j}\left|(M-j) \psi^{*}, j \psi_{\perp}^{*}\right\rangle$ where $\left|\psi^{*}\right\rangle=\sum_{i} d_{i 0}^{*}|i\rangle$. Next we expand $\left|(M-j) \psi, j \psi_{\perp}\right\rangle$ and $\left|(M-j) \psi^{*}, j \psi_{\perp}^{*}\right\rangle$ in the basis $|M, k\rangle$ according to Eq. (10), and then utilize the recurrence formula (12). Finally, we can carry out the sum over $j$ with the help of Eq. (11), resulting in 


$$
\begin{aligned}
& \left|\Phi_{\text {out }}(\psi)\right\rangle=\sum_{k=0}^{M}\left[a_{M}+b_{M}(2 k-M)\right] \cos ^{2} \frac{\vartheta}{2}|M, k\rangle \otimes|M, k\rangle+\sum_{k=0}^{M}\left[a_{M}-b_{M}(2 k-M)\right] \sin ^{2} \frac{\vartheta}{2}|M, k\rangle \otimes|M, k\rangle \\
& +e^{i \phi} \sum_{k=0}^{M} b_{M} \sin \vartheta \sqrt{(M-k)(k+1)}|M, k\rangle \otimes|M, k+1\rangle+e^{-i \phi} \sum_{k=0}^{M} b_{M} \sin \vartheta \sqrt{k(M-k+1)}|M, k\rangle \otimes|M, k-1\rangle,
\end{aligned}
$$

where the coefficients $a_{M}$ and $b_{M}$ read

$$
a_{M}=\frac{1}{\sqrt{2(M+1)}}, \quad b_{M}=\frac{\sqrt{3}}{\sqrt{2 M(M+1)(M+2)}} .
$$

The four terms on the right-hand side of Eq. (13) are proportional to the output states for the four input basis states $|01\rangle,|10\rangle,|00\rangle$, and $|11\rangle$, respectively. Consequently, it is easy to prove that the transformation $\left|\psi, \psi_{\perp}\right\rangle \rightarrow\left|\Phi_{\text {out }}(\psi)\right\rangle$ preserves scalar products, hence is unitary.

Let us now calculate the fidelity of the clones. We can see from Eq. (8) that the cloning machine preserves the symmetry of the input state $\left|\psi, \psi_{\perp}\right\rangle$, so the clones of both states $|\psi\rangle$ and $\left|\psi_{\perp}\right\rangle$ have the same fidelity. This state-independent single-qubit fidelity can be obtained by summing a series,

$$
F_{\perp}(M)=\sum_{j=0}^{M} \frac{M-j}{M} \alpha_{j, M}^{2} .
$$

After some algebra, we arrive at the expression

$$
F_{\perp}(M)=\frac{1}{2}\left(1+\sqrt{\frac{M+2}{3 M}}\right) .
$$

We are now able to compare this fidelity to that of the optimal cloner for a pair of identical qubits $F_{||}(M)$ : for $M<6$, we have $F_{||}(M)>F_{\perp}(M)$, while $F_{||}(6)=F_{\perp}(6)$ and $F_{\perp}(M)>F_{||}(M)$ for $M>6$. Thus, the cloner (8) outperforms the standard cloner for $M>6$. We note also that for $M \rightarrow \infty$, the fidelity $F_{\perp}(M)$ tends to the optimal measurement fidelity $F_{\perp}$, as expected.

The optimality of the cloner can be proved with the help of techniques adapted from the theory of semidefinite programming [12]. We observe that the trace of Lagrange multiplier $\lambda$ provides an upper bound on the achievable fidelity. If $\lambda \otimes \mathbb{1}_{\mathcal{K}}-A \geq 0$ then it holds for any $\chi$ that $\operatorname{Tr}_{\mathcal{H}, \mathcal{K}}\left[\chi \lambda \otimes \mathbb{1}_{\mathcal{K}}\right] \geq \operatorname{Tr}_{\mathcal{H}, \mathcal{K}}[\chi A]$. It follows from the unitarity constraint $\operatorname{Tr}_{\mathcal{K}}[\chi]=\mathbb{1}_{\mathcal{H}}$ that $\operatorname{Tr}_{\mathcal{H}, \mathcal{K}}\left[\chi \lambda \otimes \mathbb{1}_{\mathcal{K}}\right]=\operatorname{Tr}_{\mathcal{H}}[\lambda]$ does not depend on $\chi$. Thus it holds that $\operatorname{Tr}_{\mathcal{H}}[\lambda] \geq \operatorname{Tr}_{\mathcal{H}, \mathcal{K}}[\chi A]$. From the numerical solution of Eqs. (7) we have in basis $|00\rangle,|11\rangle,|01\rangle,|10\rangle$,

$$
\lambda=\frac{F_{\perp}(M)}{6}\left(\begin{array}{cccc}
1 & 0 & 0 & 0 \\
0 & 1 & 0 & 0 \\
0 & 0 & 2 & -1 \\
0 & 0 & -1 & 2
\end{array}\right) .
$$

The block-diagonal matrix $\lambda \otimes \mathbb{1}_{\mathcal{K}}-A$ is positive semidefinite and has three different eigenvalues which read $\mu_{1}=$ $\frac{1}{12} \sqrt{\frac{M+2}{3 M}}, \mu_{2}=\frac{1}{3} \sqrt{\frac{M+2}{3 M}}$, and $\mu_{3}=0$. Since the upper bound $\operatorname{Tr}_{\mathcal{H}}[\lambda]=F_{\perp}(M)$ is saturated by our cloning machine, we conclude that our cloner is optimal.

In the rest of this paper, we will show that the cloning transformation (8) can be implemented probabilistically via stimulated parametric down-conversion. The experimental setup under consideration is shown in Fig. 1. This scheme is a straightforward extension of the setup suggested by Simon et al. [9] where the qubits are represented by the polarization state of photons. We can identify $|0\rangle$ with vertical polarization and $|1\rangle$ with horizontal polarization states. In optical parametric downconversion, a 'blue' photon can split into a pair of 'red' photons. Traditionally, these daughter photons are referred to as signal and idler, respectively. In our setup, three nonlinear crystals $C_{1}, C_{2}, C_{3}$ are pumped by a strong laser beam. In crystals $C_{1}$ and $C_{2}$, pairs of photons can be produced, so we can verify the presence of signal photons by detecting the idler photons emerging from $C_{1}$ and $C_{2}$. If a single idler photon is detected on each side, then we have one signal photon in each beam. The states of these two photons can be manipulated with the help of phase shifters and polarization rotators in order to prepare the desired input state $\left|\psi, \psi_{\perp}\right\rangle$. The two photons then feed the signal and idler modes of a third nonlinear crystal $C_{3}$, where $M$ clones are generated due to the stimulated parametric down-conversion.

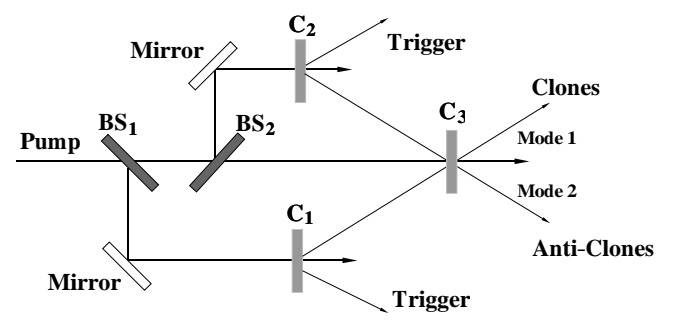

FIG. 1: Setup for the cloning of orthogonal qubits via stimulated parametric down-conversion. For a detailed description, see text.

In the limit of strong coherent pumping, the effective Hamiltonian describing the interaction in $C_{3}$ can be written as follows [9],

$$
H=i \hbar g\left(a_{V 1}^{\dagger} a_{H 2}^{\dagger}-a_{H 1}^{\dagger} a_{V 2}^{\dagger}\right)+\text { h.c. }
$$


where $a_{V 1}^{\dagger}$ and $a_{H 1}^{\dagger}$ denote bosonic creation operators for photons in the first mode with vertical (V) or horizontal $(\mathrm{H})$ polarization, and similarly $a_{V 2}^{\dagger}$ and $a_{H 2}^{\dagger}$ are creation operators for photons in the second spatial mode. The constant $g$ denotes the parametric gain. The time evolution is thus governed by the unitary transformation $U=\exp (-i H t / \hbar)$. With the help of the disentangling theorem, we can write the operator $U$ in a factorized form

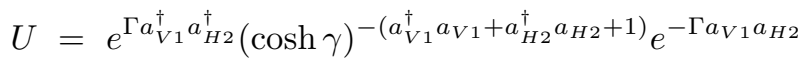

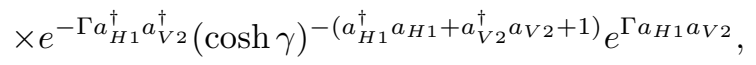

where $\gamma=g t$ and $\Gamma=\tanh \gamma$. The Hamiltonian (17) has the important property of being invariant under general simultaneous SU(2) transformations on the polarization vectors $\left(a_{V}, a_{H}\right)$ for modes 1 and 2 [9]. It is thus sufficient to consider the evolution of a basis state $|1\rangle_{V 1}|0\rangle_{H 1}|0\rangle_{V 2}|1\rangle_{H 2}$ (a single vertically polarized photon in mode 1 and a single horizontally polarized photon in mode 2) which represents the input state $\left|\psi, \psi_{\perp}\right\rangle \equiv$ $|01\rangle$. Making use of the factorized form of $U$, we obtain the state at the output of the crystal $C_{3}$ in the form

$$
\begin{aligned}
& \sum_{M=0}^{\infty} \Gamma^{M-1}\left(1-\Gamma^{2}\right) \sum_{j=0}^{M}(-1)^{j}\left[(M-j)\left(1-\Gamma^{2}\right)-\Gamma^{2}\right] \\
& \times|M-j\rangle_{V 1}|j\rangle_{H 1}|j\rangle_{V 2}|M-j\rangle_{H 2}
\end{aligned}
$$

where $|k\rangle_{l}$ with $l=V 1, H 1, V 2, H 2$ denote the usual Fock states. For a fixed number $M$ of photons in each mode 1 and 2, the output state (18) closely resembles the output state of the universal cloning machine (8) with the coefficients $\alpha_{j, M}(\Gamma) \approx\left[(M-j)\left(1-\Gamma^{2}\right)-\Gamma^{2}\right](-1)^{j}$. If we measure the number of photons in mode 2 and detect $M$ photons, then we know that $M$ photons representing $M$ approximate clones of the input qubit $|\psi\rangle$ are present in mode 1 . In order to calculate the fidelity of these clones, we insert the properly normalized $\alpha_{j, M}(\Gamma)$ into formula (14). After some algebra, we obtain

$$
F(M, y)=\frac{3 y^{2}-2 y(2 M+1)+\frac{3}{2} M(M+1)}{6 y^{2}-6 M y+M(2 M+1)}
$$

where we have introduced $y=\Gamma^{2} /\left(1-\Gamma^{2}\right) \equiv \sinh ^{2} \gamma$ for notational convenience. The cloning fidelity thus depends on the parametric gain $\gamma$, so we must optimize this gain in order to achieve the highest possible fidelity. Upon solving $\frac{\partial F(M, y)}{\partial y}=0$ for $y$, we find that

$$
y_{\mathrm{opt}}=\frac{M}{2}-\frac{1}{2} \sqrt{\frac{M(M+2)}{3}} .
$$

By inserting $y_{\text {opt }}$ into Eq. (19), we recover the optimal fidelity (15). Furthermore, it can be verified by direct calculation that with the optimal gain, the postselected $M$-photon state at the output of the crystal $C_{3}$ coincides with the output of the cloning machine (8).

This approach of cloning based on down-conversion can be further extended to the approximate realization of the general cloning transformation $|\psi\rangle^{\otimes N}\left|\psi_{\perp}\right\rangle^{\otimes N^{\prime}} \rightarrow|\psi\rangle^{\otimes M}$. For $N^{\prime}=1$, we have been able to derive the optimal fidelity for any $N$ and $M \geq N$ by a similar calculation,

$$
F_{\perp}(N, M)=\frac{N+1}{N+3}+\frac{3(N-1)+\sqrt{P /(N+2)}}{2 M(N+3)}
$$

with $P=(N-1)\left(N^{2}-15 N-18\right)+8 M(N+1)(M+3-N)$. It can be checked that there is again a value of $M$ above which this cloner outperforms the standard $(N+1) \rightarrow M$ cloner. For large $N$, however, the advantage becomes marginal.

In summary, we have designed a universal cloning machine for orthogonal qubit pairs, and have shown that it achieves a higher fidelity for $M>6$ than the standard cloner for identical qubits. We conclude that the advantage of orthogonal qubits over identical qubits that was discovered in the context of measurement also extends to cloning.

N.J.C. is grateful to Christoph Simon for helpful discussions. J.F. acknowledges support from the grant No LN00A015 of the Czech Ministry of Education. S.I. is supported by a fellowship from the Belgian FRIA foundation. N.C and S.M. acknowledge funding by the European Union under project IST-FET-EQUIP.
[1] W.K. Wootters and W.H. Zurek, Nature (London) 299, 802 (1982); D. Dieks, Phys. Lett. 92A, 271 (1982).

[2] V. Bužek and M. Hillery, Phys. Rev. A 54, 1844 (1996); N. Gisin and S. Massar, Phys. Rev. Lett. 79, 2153 (1997); R.F. Werner, Phys. Rev. A 58, 1827 (1998); D. Bruss, A. Ekert, and C. Macchiavello, Phys. Rev. Lett. 81, 2598 (1998).

[3] V. Bužek and M. Hillery, Phys. Rev. Lett. 81, 5003 (1998); N.J. Cerf, J. Mod. Opt. 47, 187 (2000); S.L. Braunstein, V. Bužek, and M. Hillery, Phys. Rev. A 63, 052313(2001).

[4] N.J. Cerf, A. Ipe, and X. Rottenberg, Phys. Rev. Lett. 85, 1754 (2000); S.L. Braunstein, N.J. Cerf, S. Iblisdir,
P. van Loock, and S. Massar, Phys. Rev. Lett. 86, 4938 (2001); J. Fiurášek, Phys. Rev. Lett. 86, 4942 (2001).

[5] N. Gisin and S. Popescu, Phys. Rev. Lett. 83, 432 (1999).

[6] S. Massar, Phys. Rev. A 62, 040101(R) (2000).

[7] N.J. Cerf and S. Iblisdir, Phys. Rev. A 64032307 (2001).

[8] N.J. Cerf and S. Iblisdir, e-print quant-ph/0102077.

[9] C. Simon, G. Weihs, and A. Zeilinger, Phys. Rev. Lett. 84, 2993 (2000).

[10] J. Fiurášek, e-print quant-ph/0105124.

[11] N.Ja. Vilenkin and A.U. Klimik, Representation of Lie Groups and Special Functions (Kluwer, Dordrecht, 1991).

[12] K. Audenaert and B. de Moor, e-print quant-ph/0109155. 